The inequality is certainly true for $k=0$. Hence, assuming that it holds for $k$, we have

$$
\begin{aligned}
f_{i}^{(k+1)} & =\operatorname{Min}_{i \neq i}\left[t_{i i}+f_{i}^{(k)}\right] \leq \operatorname{Min}_{j \neq i}\left[t_{i j}+f_{i}\right] \\
& \leq f_{i} .
\end{aligned}
$$

It follows that the sequence $\left\{f_{i}^{(k)}\right\}$ converges to $\left\{f_{i}\right\}$ as $k \rightarrow \infty$, furnishing the desired monotone convergence. Once again, only a finite number of iterations will be required. It is to be expected that the first method will converge more rapidly.

\title{
REFERENCES
}

1. R. Bellman, Dynamic Programming, Princeton University Press, 1957

2. R. Bellman, The Theory of Dynamic Programming, Bull. Amer. Math. Soc., 60, 503-515 (1954)

\section{ON THE DAMPED OSCILLATIONS EQUATION WITH VARIABLE COEFFICIENTS*}

BY E. V. IAITONE (University of California, Berkeley)

A useful upper bound can be given for the oscillatory solutions of the second-order linear differential equation with continuous differentiable coefficients

$$
u^{\prime \prime}(t)+p(t) u^{\prime}(t)+q(t) u(t)=0
$$

which commonly occurs in vibration studies and airplane or missile dynamics.

The solutions of Eq. (1) are oscillatory whenever

$$
\phi(t)=\left(q-p^{2} / 4-p^{\prime} / 2\right) \geqq m^{2}>0
$$

see Bellman [1] or Kamke [2], and for this case it will be shown that as long as $\phi$ is monotonic the solutions are bounded in the following manner:

$$
|u(t)| \leqq \frac{1}{m}\left\{\phi(0) u(0)^{2}+\left[u^{\prime}(0)+p(0) u(0) / 2\right]^{2}\right\}^{1 / 2} \exp \left(-\int_{0}^{t} p / 2 d t\right) .
$$

This result can be derived by introducing the unique transformation, see Bellman [1] or Kamke [2]

$$
u=v \exp \left(-\int_{0}^{t} p / 2 d t\right)
$$

which preserves the same zeros in the oscillatory solutions. Then Eq. (1) is transformed to

$$
v^{\prime \prime}(t)+\phi(t) v(t)=0,
$$

*Received February 5, 1957. 
where $\phi$ is defined by Eq. (2). The inequality $\phi \geqq m^{2}>0$ insures that all the solutions are oscillatory, see Bellman [1] or Kamke [2], also it is known that if in addition $\phi$ is monotonic, then the amplitudes of $v$ also vary monotonically, increasing when $\phi$ is decreasing and vice-versa.

Then the function

$$
F(t)=\phi v^{2}+v^{2}
$$

is introduced, see Kamke [2], in order to develop the inequality

$$
\frac{F^{\prime}(t)}{F(t)}=\frac{2 \phi v v^{\prime}+\phi^{\prime} v^{2}+2 v^{\prime} v^{\prime \prime}}{\phi v^{2}+v^{\prime 2}}=\frac{\phi^{\prime} v^{2}}{\phi v^{2}+v^{2}} \leqq \frac{\phi^{\prime}(t)}{\phi(t)}
$$

which will be used to derive Eq. (3).

Case I, $\phi$ monotonically decreasing.

Since $\phi^{\prime}<0$, therefore $F^{\prime}=\phi^{\prime} v^{2} \leqq 0$ so that

$$
F(0) \geqq F(t) \geqq \phi(t) v(t)^{2} \geqq m^{2} v(t)^{2} .
$$

Consequently

$$
|v(t)| \leqq \frac{1}{m} F(0)^{1 / 2}=\frac{1}{m}\left[\phi(0) v(0)^{2}+v^{\prime}(0)^{2}\right]^{1 / 2}
$$

which transforms to Eq. (3) upon introducing Eq. (4).

Case II, $\phi$ monotonically increasing.

Since $\phi^{\prime}>0$, therefore $\phi(t)>\phi(0) \geqq m^{2}$ and $F^{\prime}=\phi^{\prime} v^{2} \geqq 0$ so that

$$
F(t) \geqq F(0) \geqq \phi(0) v(0)^{2} \geqq m^{2} v(0)^{2} .
$$

Consequently Eq. (7) may be integrated to

$$
\ln \frac{\phi(t)}{\phi(0)} \geqq \ln \frac{F(t)}{F(0)} \geqq \ln \frac{\phi(t) v(t)^{2}}{\phi(0) v(0)^{2}+v^{\prime}(0)^{2}} .
$$

This shows that if $v^{2}<1$ and

$$
\phi(0)>F(0)>0
$$

then Eq. (11) again yields Eq. (9), and similarly, Eq. (3). Now $F>0$ for $\phi>0$ since $v$ and $v^{\prime}$ cannot be simultaneously zero, however $\phi>F$ only for sufficiently small initial displacements from equilibrium so that $v^{2}<1$ and $v^{\prime 2}<\left(1-v^{2}\right) \phi \leqq \phi$.

Consequently the bound provided for Eq. (1) by Eq. (3) is always valid when $\phi\left(\geqq m^{2}>0\right)$ is monotonically decreasing, but is only valid for sufficiently small initial values of $u$ and $u^{\prime}$ so that Eq. (12) is satisfied when $\phi$ is monotonically increasing. This latter requirement is satisfied whenever

$$
\phi(0)>\left\{\phi(0) u(0)^{2}+\left[u^{\prime}(0)^{2}+p(0) u(0) / 2\right]^{2}\right\}
$$

in which case Eq. (3) could be replaced by

$$
|u(t)| \leqq \frac{1}{m} \phi(0)^{1 / 2} \exp \left(-\int_{0}^{t} p / 2 d t\right)
$$


or in the usual situation wherein $\phi(0)=m^{2}$ when $\phi$ is monotonically increasing, by

$$
|u(t)| \leqq \exp \left(-\int_{0}^{t} p / 2 d t\right) .
$$

The limiting behavior of Eq. (5) as $t \rightarrow \infty$, see Bellman [1] or Kamke [2], shows that in both cases the solutions of Eq. (1) can be written for $t \rightarrow \infty$ as

$$
u \rightarrow C_{1} \sin \left(a t+C_{2}\right) \exp \left(-\int_{0}^{t} p / 2 d t\right),
$$

whenever $\phi(t) \rightarrow a^{2}\left(\geqq m^{2}>0\right)$ as $t \rightarrow \infty$ and

$$
\int_{0}^{\infty}\left|\frac{\phi(t)}{a^{2}}-1\right| d t<\infty .
$$

Application to dynamic stability analysis of a missile. As a typical application of the upper bound provided by Eq. (3) let us consider the case of a missile having a nearly vertical trajectory with no control forces or thrust misalignment. In this case the equations presented in [3], which define the variation of the small incremental angle of attack $\alpha$, during constant acceleration, may be written as

$$
\alpha^{\prime \prime}(t)+p(t) \alpha^{\prime}(t)+q(t) \alpha(t)=0 .
$$

Therefore as long as $p$ and $q$ are such that the $\phi$ defined by Eq. (2) is monotonic, the upper bound of $\alpha$ can be determined. This is generally true for constant atmospheric density since the forward velocity $U$ is continually increasing while the rate of acceleration is generally small. However if the magnitude of the acceleration is sufficiently large, or the height above the earth's surface is rapidly varying, then it is necessary to transform the independent variable from the time to the distance along the trajectory, as in [3], before Eq. (3) can be applied.

A particularly interesting case occurs for the vertical entry of a missile into the earth's atmosphere at hypersonic speeds. Then the ratio of the acceleration to the velocity squared is nearly constant so that in terms of $\chi$, the non-dimensional distance along the trajectory normal to the earth's surface, the variation of the small incremental angle of attack may be approximated to the first order by

$$
\alpha^{\prime \prime}(\chi)+b \rho(\chi) \alpha^{\prime}(\chi)+c \rho(\chi) \alpha(\chi)=0,
$$

where $b$ and $c$ are constants and $\rho$ is the mass density of the air.

Consequently, Eq. (2) becomes

$$
\phi(\chi)=\left[c \rho-(b \rho / 2)^{2}-b \rho^{\prime} / 2\right]
$$

and it is easily shown that $\phi(\chi)$ is always bounded and monotonically increasing for any physically possible variation in air density as long as $\phi(0)>0$ for the constant mass missile at hypersonic speeds.

For example, the usual exponential approximation to the density variation may be written in terms of $\rho_{\infty}$, the sea-level mass density of the air, as

$$
\rho(\chi)=\rho_{\infty}\left\{1-\exp \left[-\beta\left(\chi+\chi_{0}\right)\right]\right\}
$$

so that $\phi$ is monotonically increasing and bounded by

$$
0<\phi(0) \leqq \phi(\chi)<\left[c \rho_{\infty}-\left(b \rho_{\infty} / 2\right)^{2}-b \beta \rho_{\infty} / 2\right]
$$


since

$$
\begin{aligned}
\rho_{\infty} & \ll 1 \mathrm{lb} . \sec ^{2} \mathrm{ft}^{-4} \\
0 & <\beta \ll 1 \mathrm{ft}^{-1},
\end{aligned}
$$

for any physically possible atmosphere.

Therefore $\alpha(\chi)$ is oscillatory as long as $\phi(0)>0$, and the upper bound is defined by Eq. (3) as long as

$$
\phi(0)>\left\{\phi(0) \alpha(0)^{2}+\left[\alpha^{\prime}(0)^{2}+b \rho(0) \alpha(0) / 2\right]^{2}\right\},
$$

that is, provided Eq. (13) is satisfied.

\section{REFERENCES}

1. R. Bellman, Stability theory of differential equations, McGraw-Hill, N. Y., 1953, Chap. 6

2. E. Kamke, Differentialgleichungen, vol. I, Gewöhnliche Differentialgleichungen, 3d ed., Chelsea Publish: ing Co., 1948, pp. 119-138

3. T. W. Oswald, Dynamic behaviour during accelerated fight with particular application to missile launching, J. Aeronaut. Sci. 23, 781-791 (1956) 